\title{
Presence of free triiodothyronine and free thyroxine in thyroid follicles may be correlated with the quick secretion of thyroid hormones under certain physiological conditions
}

\author{
HAIHONG SHI ${ }^{1}$, WANRONG LIN ${ }^{2}$, BO LIANG ${ }^{3}$, HUIYAO CAI $^{3}$, \\ QINGYAN CAI ${ }^{3}$, YAXIONG $\mathrm{SHI}^{3}$ and HUIBIN HUANG ${ }^{3}$ \\ ${ }^{1}$ Department of Oncological Surgery, The Second Affiliated Hospital of Fujian Medical University; \\ ${ }^{2}$ The Second Clinical Medical College of Fujian Medical University; ${ }^{3}$ Department of Endocrinology, \\ The Second Affiliated Hospital of Fujian Medical University, Quanzhou, Fujian 362000, P.R. China
}

Received October 26, 2015; Accepted February 2, 2016

DOI: $10.3892 /$ br.2016.596

\begin{abstract}
Thyroid cells are polarized and the follicle structure, consisting of follicle epithelial cells, is a prerequisite for thyroid hormone synthesis. However, a reliable in vitro model simulating thyroid function is not currently available. To the best of our knowledge, the present study reports for the first time a simulated follicle by inoculation of human thyroid cells on the filter in a Transwell plate to maintain the polarity of thyroid cells. The iodine uptake was analyzed by arsenic and cerium catalysis spectrophotometry, as well as the secretion of free triiodothyronine (FT3) and free thyroxine (FT4) by direct chemiluminescence. The data showed that thyroid cells growing in the Transwell chamber synthesized and secreted FT3 and FT4, while the monolayer cells directly seeded in the 6-well-plate did not produce these two thyroid hormones. Regarding the iodine uptake, cells in the Transwell chamber demonstrated a markedly higher capability than the monolayer cells. The data proved that the polarity of thyroid cells could be restored using the Transwell plate, which was critical for iodine uptake and thyroid hormone synthesis. The presence of FT3 and FT4 in follicles may be correlated with the quick secretion of thyroid hormones under certain physiological conditions.
\end{abstract}

\section{Introduction}

The mammalian thyroid gland consists of numerous follicular structures, which are known as follicles (1). Each follicle

Correspondence to: Dr Huibin Huang, Department of Endocrinology, The Second Affiliated Hospital of Fujian Medical University, 34 North Zhongshan Road, Quanzhou, Fujian 362000, P.R. China

E-mail: huibinhuang@aliyun.com

Key words: thyroid follicle, free triiodothyronine, free thyroxine, iodine uptake is constructed by abundant epithelial cells arranged as an epithelium (2). A follicle lumen surrounded by these polarized epithelial cells represents the most fundamental thyroid unit, where the synthesis and secretion of hormones, as well as iodine uptake, are processed (3). The normal thyroid functions will be compromised if the intact follicle structure is broken. The monolayer thyroid cells do not possess these functions due to loss of polarity and absence of follicular structure. The unavailability of a functional thyroid model significantly impedes the progression of scientific research on the regulatory mechanisms of thyroid function. In virtue of the Transwell plate, the natural structure of a thyroid follicle was simulated in the present study by inoculating thyroid Nthy-ori3-1 cells onto the chamber filter, and restoring the normal thyroid cellular function of secreting thyroid hormone.

\section{Materials and methods}

Materials. The human thyroid cell line Nthy-ori-3-1 was purchased from the European Collection of Authenticated Cell Cultures (Salisbury, UK). Potassium iodide (KI) and thyroid-stimulating hormone (TSH) were purchased from Sigma-Aldrich (St. Louis, MO, USA). All the reagents and plates for cell culture were products of Corning, Inc. (Corning, NY, USA). The chemiluminescence kits for the detection of free triiodothyronine (FT3) and free thyroxine (FT4) were from Siemens AG (Munich, Germany). The iodine automatic detector was purchased from Wentesi Health Technology, Ltd. (Hubei, China).

Cell culture. Nthy-ori-3-1 cells were cultured in RPMI-1640 medium and $10 \%$ fetal bovine serum (FBS) in a $37^{\circ} \mathrm{C}$ incubator with $5 \% \mathrm{CO}_{2}$. The culture medium was replaced every 2 days and the cells were routinely passaged every 7 days.

Establishment of thyroid follicle model in vitro. The in vitro follicle model is schematically illustrated in Fig. 1. The polycarbonate filter membrane was firstly coated with $0.3 \mathrm{mg} / \mathrm{ml}$ collagen followed by inoculation of the Nthy-ori-3-1 cells $\left(\sim 1.5 \times 10^{6}\right.$ cells/filter). In the lower chamber, 


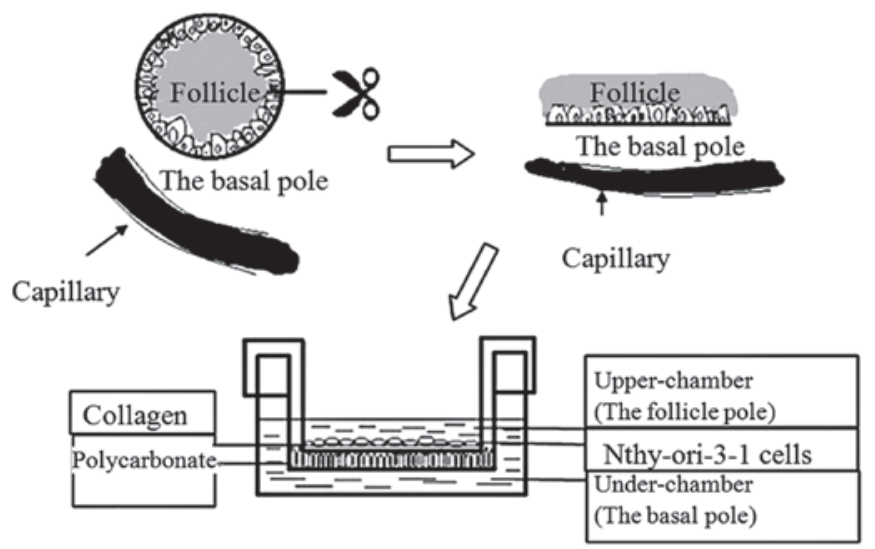

Figure 1. A schematic illustration of the follicle model. The polycarbonate filter membrane was firstly coated with $0.3 \mathrm{mg} / \mathrm{ml}$ collagen, followed by inoculation of the Nthy-ori-3-1 cells. In the lower chamber, $2 \mathrm{ml}$ RPMI-1640 medium with 10\% FBS, 1 mIU/1 thyroid-stimulating hormone and $330 \mu \mathrm{g} / 1$ potassium iodide was added, while the upper chamber was filled with $0.8 \mathrm{ml}$ RPMI-1640 medium with $10 \%$ FBS. The upper and lower chambers were completely separated subsequent to the cells reaching $100 \%$ confluence. The upper chamber acted as the follicular lumen, the interface between the cell and upper chamber served as the apical surface and the cell-lower chamber contact area was similar to the basal pole. FBS, fetal bovine serum.
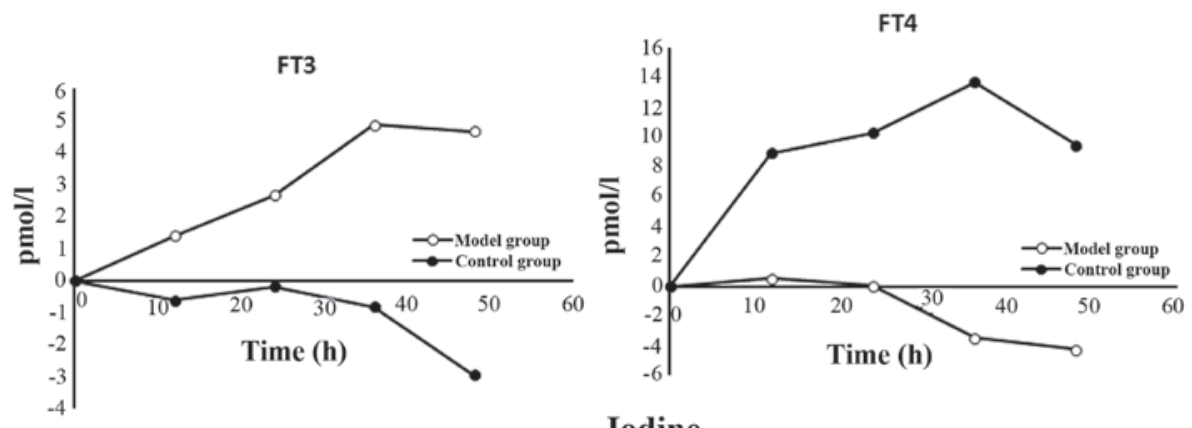

Iodine

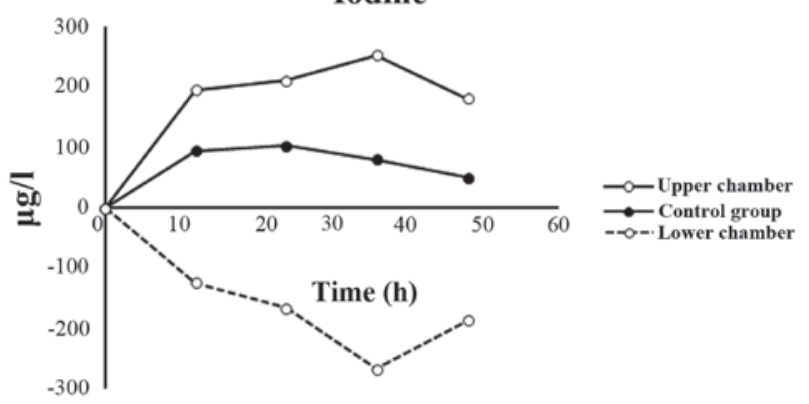

Figure 2. Synthesis of FT3 and FT4, and iodine uptake changes in a time-dependent manner. The synthesis of thyroid hormones and iodine uptake in the upper chamber increased in a time-dependent manner and reached a peak value at $36 \mathrm{~h}$; however, in the monolayer cells, the amount of FT3 and FT4 in the culture medium decreased, and the iodine uptake reached a peak value at $12 \mathrm{~h}$. FT3, free triiodothyronine; FT4, free thyroxine.
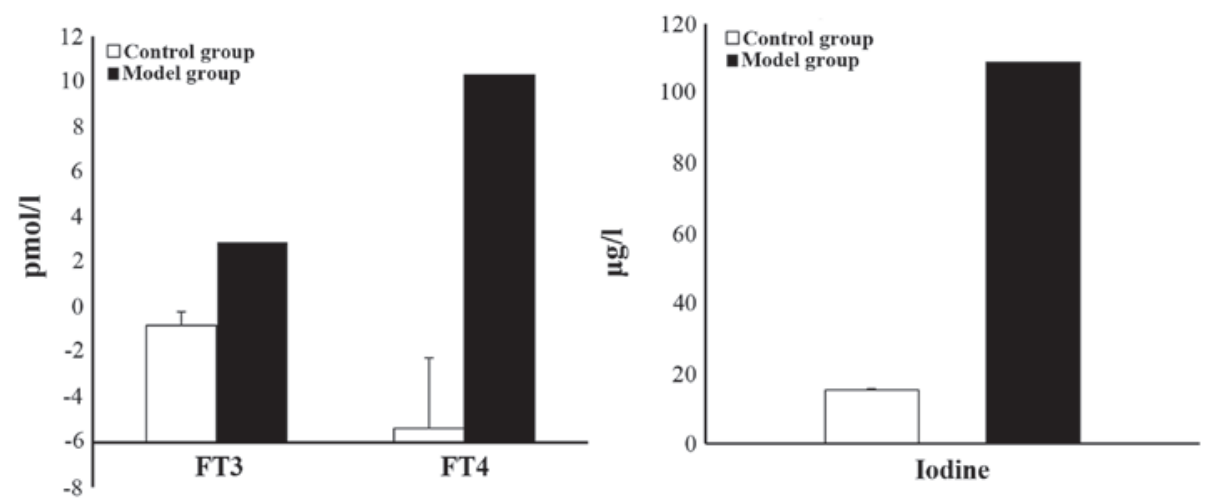

Figure 3. Thyroid cells cultivated in the Transwell plates produce FT3 and FT4, and take up iodine. The monolayer cells were almost not able to synthesize FT3 and FT4, and possessed a limited capability of taking up iodine. In comparison, the thyroid cells growing in the Transwell chamber demonstrated a higher capability of producing FT3 and FT4, and a stronger capacity of iodine uptake. 
$2 \mathrm{ml}$ of RPMI-1640 medium with 10\% FBS, 1 mIU/1 TSH and $330 \mu \mathrm{g} / 1 \mathrm{KI}$ was added, while the upper chamber was filled with $0.8 \mathrm{ml}$ RPMI-1640 medium without $10 \%$ FBS. The liquid level between the upper and lower chambers was aligned. As a control, the monolayer cells, with an identical number of cells, were cultured in a collagen-coated plate, and were maintained in the same medium as used in the lower chamber. The culture medium was replaced respectively when the cells reached complete confluence, and the cells were continuously cultured for an extra 36 or $48 \mathrm{~h}$. Following this, the culture medium was subjected to analysis. The iodine uptake was determined by arsenic and cerium catalysis spectrophotometry and the secretion of FT3 and FT4 was detected by direct chemiluminescence.

Statistical analysis. SPSS software 19 (IBM. Corp., Armonk, NY, USA) was employed for statistical analysis. Statistical significance among the groups was assessed using unpaired Student's t-test. All the data were expressed as mean \pm standard error.

\section{Results}

Thyroid hormone synthesis and iodine uptake. The synthesis of thyroid hormones and iodine uptake in the upper chamber increased in a time-dependent manner and reached a peak value at $36 \mathrm{~h}$; however, in the monolayer cells, the amount of FT3 and FT4 in the culture medium decreased, and the iodine uptake reached a peak value at $12 \mathrm{~h}$ (Fig. 2).

The monolayer cells were almost not able to synthesize FT3 and FT4 and only possessed a limited capability of iodine uptake; $15.13 \pm 8.78 \mu \mathrm{g}$, (Fig. 3 and Table I). In comparison, thyroid cells growing in the Transwell chamber demonstrated a higher capability of producing FT3 and FT4, which were $2.89 \pm 0.21$ and $10.35 \pm 0.14$ pmol, respectively, and stronger capacity of iodine uptake, which was 109.52 $\pm 3.18 \mu \mathrm{g}$ (Fig. 3 and Table I), indicating that the cells growing in the Transwell chamber simulated thyroid function and demonstrated incomparable advantages compared to the monolayer cells (Table I).

\section{Discussion}

The thyroid follicle is the fundamental unit where iodine uptake and thyroid hormone synthesis occurs. The intact follicular structure is a prerequisite for thyroid gland exercising normal function. All the models that are feasible for thyroid function research must rebuild the follicle structure. Previously, thyroid tissue sections were often used as a research model (4). Although this model had follicles and possessed the ability of iodine uptake and synthesis of hormones, other cell types mixed in the model would influence the experimental outcomes; furthermore, observing the phenotype and extracting the components in follicles is challenging. Previous studies have attempted to replace tissue culture with primary thyroid epithelium or cell lines (5-9). All these cells usually grew in a monolayer. Using gel and serum could aid with the formation of follicle-like structures; however, this method did not overcome the barriers of follicular component isolation and observation of the follicle; and additionally, follicle-like
Table I. Thyroid hormone synthesis and iodine uptake by monolayer cells in a 6-well or Transwell plate.

\begin{tabular}{lllc}
\hline Groups & FT3, pmol & FT4, pmol & Iodine, $\mu \mathrm{g}$ \\
\hline Monolayer cells & $-0.8 \pm 0.61$ & $-5.37 \pm 3.13$ & $15.13 \pm 8.78$ \\
Transwell group & $2.89 \pm 0.21^{\mathrm{a}}$ & $10.35 \pm 0.14^{\mathrm{a}}$ & $109.52 \pm 3.18^{\mathrm{a}}$ \\
\hline
\end{tabular}

${ }^{\mathrm{a}} \mathrm{P}<0.05(\mathrm{n}=3)$. FT3, free triiodothyronine; FT4, free thyroxine.

structures could only be sustained for a short time span. All these defects significantly limited their application in the study of thyroid function. Inversely, there were advantages when using monolayer cells as they can simulate the function of the follicle structure.

In the present study, the human thyroid cell line Nthy-ori3-1 and Transwell plates were used to simulate thyroid structure, and the function of this model was primarily validated. Following inoculation on the filter membrane in the upper chamber, thyroid cells grew polarized in the monolayer and closely attached to the culture medium in the lower chamber, which were filled with TSH and iodine in addition to the complete culture medium. The upper and lower chambers were completely separated subsequent to the cells reaching $100 \%$ confluence. The upper chamber acted as the follicular lumen, the interface between cell and upper chamber served as the apical surface and the cell-lower chamber contact area was similar to the basal pole (Fig. 1). Cells took up nutrient materials from the lower chamber for synthesizing thyroglobulin (Tg) and thyroid hormones, which were lately released into the upper chamber. The in vitro model established was an open system, and was barrier-free for extraction of follicular components, as well as the application of various treatments. This model showed unique advantages for investigating the follicular components, as well as their regulatory roles in mediating thyroid functions.

Postgård et al (10) previously built a similar model using porcine primary thyroid cells; however, porcine primary thyroid cells were not feasible for long-term cultivation, and the monolayer cells were mixed with the intact follicle on the filter membrane when the cells were seeded at higher density, so it was not an ideal option for elucidating thyroid function. In the current model, the human-derived thyroid cell line Nthy-ori-3-1 only grew in a monolayer manner, which benefited the reconstruction of the functional thyroid model in vitro.

For verifying whether the model established would function correctly, several assays were conducted to probe the secretion of FT3 and FT4, as well as the capability of iodine uptake. The data showed that the amount of FT3 and FT4 in the upper chamber was markedly higher than those from monolayer cells. To be precise, the levels of FT3 and FT4 in the monolayer cells were below the detection threshold, indicating monolayer cells not only failed to synthesize hormones, but also consumed the original hormones. Based on a previous study, follicular T3 and T4 were coupled with Tg prior to uptake and hydrolysis by thyroid cells (11); therefore, FT3 and FT4 were not detected in the follicles. However, a certain amount of free 
thyroid hormones existed in the present model, which could explain the temporary thyrotoxia caused by FT3 and FT4 leakage in various thyroiditis. Generally, thyroid cells require time to take up T3 or T4 coupled with $\mathrm{Tg}$ and hydrolyze them prior to release in the blood. The existence of T3 and T4 in follicles may be correlated with the quick secretion of thyroid hormones under certain stimuli conditions, like cold conditions or hunger.

Due to a lack of polarity, monolayer cells cannot synthesize thyroid hormones and take up iodine. Iodine is the raw material for hormone synthesis and will be transported to and organized in follicles binding with $\mathrm{Tg}$, and this process is designated as iodine uptake. Following uptake, the intra- and extracellular iodine concentration will equilibrate if iodine cannot be transported out of the cell, which in turn impedes iodine uptake. The model built in the present study could take up iodine significantly; the iodine concentration in the upper chamber increased in a time-dependent manner, while it decreased in the lower chamber due to the uptake by thyroid cells. In the monolayer cells, the iodine concentration in the culture medium only decreased, indicating that the monolayer cells had defects in iodine uptake.

Taken together, to the best of our knowledge, the present study reports for the first time that Transwells and the human thyroid cell line can be used to create a thyroid follicle model. This model simulated thyroid follicle, synthesized thyroid hormones and iodine was taken up by the cells. The presence of FT3 and FT4 in the follicles may be correlated with the quick secretion of thyroid hormones under certain physiological conditions.

\section{Acknowledgements}

The present study was supported by the Natural Science Foundation of China (grant no. 81370886), the Natural Science Foundation of Fujian (grant no. 2012J01332), the Key Scientific Project of Fujian Province (grant no. 2014Y0017), and the Innovative Medical Research Project of Fujian Province (grant no. 2012-CXB-24).

\section{References}

1. Wang W, Ozolek JA and Rohde GK: Detection and classification of thyroid follicular lesions based on nuclear structure from histopathology images. Cytometry A 77: 485-494, 2010.

2. Mauchamp J, Mirrione A, Alquier C and Andre F: Follicle-like structure and polarized monolayer: Role of the extracellular matrix on thyroid cell organization in primary culture. Biol Cell 90: 369-380, 1998.

3. Takasu N, Ohno S, Komiya I and Yamada T: Requirements of follicle structure for thyroid hormone synthesis; cy toskeletons and iodine metabolism in polarized monolayer cells on collagen gel and in double layered, follicle-forming cells. Endocrinology 131: 1143-1148, 1992 .

4. Langer R, Burzler C, Bechtner G and Gärtner R: Influence of iodide and iodolactones on thyroid apoptosis. Influence of iodide and iodolactones on thyroid apoptosis. Evidence that apoptosis induced by iodide is mediated by iodolactones in intact porcine thyroid follicles. Exp Clin Endocrinol Diabetes 111: 325-329, 2003.

5. Toda S, Aoki S, Uchihashi K, Matsunobu A, Yamamoto M, Ootani A, Yamasaki F, Koike E and Sugihara H: Culture models for studying thyroid biology and disorders. ISRN Endocrinol 2011: 275782, 2011

6. Bernier-Valentin F, Trouttet-Masson S, Rabilloud R, Selmi-Ruby S and Rousset B: Three-dimensional organization of thyroid cells into follicle structures is a pivotal factor in the control of sodium/iodide symporter expression. Endocrinology 147: 2035-2042, 2006.

7. Liu H, Zeng Q, Cui Y, Zhao L, Zhang L, Fu G, Hou C, Zhang S, Yu L, Jiang C, et al: The role of the IRE1 pathway in excessive iodide- and/or fluoride-induced apoptosis in Nthy-ori 3-1 cells in vitro. Toxicol Lett 224: 341-348, 2014.

8. Noguchi Y, Harii N, Giuliani C, Tatsuno I, Suzuki K and Kohn LD: Thyroglobulin (Tg) induces thyroid cell growth in a concentration-specific manner by a mechanism other than thyrotropin/cAMP stimulation. Biochem Biophys Res Commun 391: 890-894, 2010.

9. Huang H, Shi Y, Lin L, Li L, Lin X, Li X and Xu D: Inhibition of thyroid-restricted genes by follicular thyroglobulin involves iodinated degree. J Cell Biochem 112: 971-977. 2011.

10. Postgård P, Himmelman J, Lindencrona U, Bhogal N, Wiberg D, Berg G, Jansson S, Nyström E, Forssell-Aronsson E and Nilsson M: Stunning of iodide transport by (131)I irradiation in cultured thyroid epithelial cells. J Nucl Med 43: 828-834, 2002.

11. Lacroix L, Pourcher T, Magnon C, Bellon N, Talbot M, Intaraphairot T, Caillou B, Schlumberger $\mathrm{M}$ and Bidart JM: Expression of the apical iodide transporter in human thyroid tissues: A comparison study with other iodide transporters. J Clin Endocrinol Metab 89: 1423-1428, 2004. 